\title{
Asymmetric Truncated Toeplitz Operators of Rank One
}

\author{
Bartosz Lanucha $^{1}$ (D)
}

Received: 17 July 2017 / Revised: 8 August 2017 / Accepted: 15 August 2017 /

Published online: 30 October 2017

(C) The Author(s) 2017. This article is an open access publication

\begin{abstract}
A truncated Toeplitz operator is a compression of the multiplication operator to a backward shift invariant subspace of the Hardy space $H^{2}$. An asymmetric truncated Toeplitz operator is a compression of the multiplication operator that acts between two different backward shift invariant subspaces of $H^{2}$. All rank-one truncated Toeplitz operators have been described by Sarason. Here, we characterize all rank-one asymmetric truncated Toeplitz operators. This completes the description given by Łanucha for asymmetric truncated Toeplitz operators on finite-dimensional backward shift invariant subspaces.
\end{abstract}

Keywords Model space - Truncated Toeplitz operator - Asymmetric truncated Toeplitz operator $\cdot$ Rank-one operator

Mathematics Subject Classification 47B32 - 47B35 · 30H10

\section{Introduction}

Denote by $H^{2}$ the Hardy space of the open unit disk $\mathbb{D}=\{z:|z|<1\}$ and let $P$ be the orthogonal projection from $L^{2}(\partial \mathbb{D})$ onto $H^{2}$.

Communicated by Stephan Ruscheweyh.

$凶$ Bartosz Łanucha

bartosz.lanucha@poczta.umcs.lublin.pl

1 Department of Mathematics, Maria Curie-Skłodowska University, Maria Curie-Skłodowska Square 1, 20-031 Lublin, Poland 
A classical Toeplitz operator $T_{\varphi}$ with symbol $\varphi \in L^{\infty}(\partial \mathbb{D})$ is defined on $H^{2}$ by

$$
T_{\varphi} f=P(\varphi f)
$$

It is clear that a Toeplitz operator with symbol from $L^{\infty}(\partial \mathbb{D})$ is bounded. Note that if $\varphi \in L^{2}(\partial \mathbb{D})$, then the above definition produces an operator $T_{\varphi}$, densely defined on $H^{\infty}=H^{2} \cap L^{\infty}(\partial \mathbb{D})$. It is known that such $T_{\varphi}$ extends boundedly to $H^{2}$ if and only if $\varphi \in L^{\infty}(\partial \mathbb{D})$.

The operators $S=T_{z}$ and $S^{*}=T_{\bar{z}}$ are called the unilateral shift and the backward shift, respectively. Obviously, $S f(z)=z f(z)$ and a simple computation reveals that

$$
S^{*} f(z)=\frac{f(z)-f(0)}{z} .
$$

A truncated Toeplitz operator is a compression of a classical Toeplitz operator to a model space. Recall, that model spaces are the closed subspaces of $H^{2}$ of the form

$$
K_{\alpha}=H^{2} \ominus \alpha H^{2}
$$

where $\alpha$ is an inner function, i.e., $\alpha \in H^{\infty}$ and $|\alpha|=1$ a.e. on $\partial \mathbb{D}$. As a consequence of the theorem of Beurling, model spaces are the typical non-trivial $S^{*}$-invariant subspaces of $H^{2}$.

Extensive study of the class of truncated Toeplitz operators began in 2007 with Sarason's paper [10]. Generalizations of these operators, the so-called asymmetric truncated Toeplitz operators, were recently introduced in $[2,4,5]$.

Let $\alpha, \beta$ be two inner functions. An asymmetric truncated Toeplitz operator $A_{\varphi}^{\alpha, \beta}$ with symbol $\varphi \in L^{2}(\partial \mathbb{D})$ is given by

$$
A_{\varphi}^{\alpha, \beta} f=P_{\beta}(\varphi f), \quad f \in K_{\alpha},
$$

where $P_{\beta}$ is the orthogonal projection from $L^{2}(\partial \mathbb{D})$ onto $K_{\beta}$. In particular, a truncated Toeplitz operator is the operator $A_{\varphi}^{\alpha}=A_{\varphi}^{\alpha, \alpha}$.

Since $P_{\beta}(\varphi f) \in K_{\beta}$ for $f \in K_{\alpha}^{\infty}=K_{\alpha} \cap H^{\infty}$ and $K_{\alpha}^{\infty}$ is dense in $K_{\alpha}$, the operator $A_{\varphi}^{\alpha, \beta}$ is densely defined. It is known that the boundedness of $\varphi$ is not necessary for $A_{\varphi}^{\alpha, \beta}$ to extend boundedly to $K_{\alpha}$.

Put

$$
\mathscr{T}(\alpha, \beta)=\left\{A_{\varphi}^{\alpha, \beta}: \varphi \in L^{2}(\partial \mathbb{D}) \text { and } A_{\varphi}^{\alpha, \beta} \text { is bounded }\right\}
$$

and $\mathscr{T}(\alpha)=\mathscr{T}(\alpha, \alpha)$.

Sarason showed that the truncated Toeplitz operators of rank one are generated (in a sense to be explained below) by functions of the form

$$
k_{w}^{\alpha}(z)=\frac{1-\overline{\alpha(w)} \alpha(z)}{1-\bar{w} z}
$$


and

$$
\widetilde{k}_{w}^{\alpha}(z)=\frac{\alpha(z)-\alpha(w)}{z-w} .
$$

The function $k_{w}^{\alpha}$, called the reproducing kernel, is the kernel function for the point evaluation functional $f \mapsto f(w)$, which for $w \in \mathbb{D}$ is bounded on $K_{\alpha}$. In other words, $f(w)=\left\langle f, k_{w}^{\alpha}\right\rangle$ for every $f \in K_{\alpha}$ and each $w \in \mathbb{D}$. It is easy to check that $k_{w}^{\alpha}=P_{\alpha} k_{w}$, where $k_{w}(z)=(1-\bar{w} z)^{-1}$.

The function $\widetilde{k}_{w}^{\alpha}$ is the image of the reproducing kernel $k_{w}^{\alpha}$ under the map

$$
C_{\alpha} f(z)=\alpha(z) \overline{z f(z)}, \quad|z|=1 .
$$

One can verify that $C_{\alpha}$ is an antilinear and isometric involution on $L^{2}(\partial \mathbb{D})$ which preserves $K_{\alpha}$. The map $C_{\alpha}$ is called a conjugation and, since $\widetilde{k}_{w}^{\alpha}=C_{\alpha} k_{w}^{\alpha}$, the function $\widetilde{k}_{w}^{\alpha}$ is often called the conjugate kernel.

It should be noted that the functional $f \mapsto f(w)$ is bounded on $K_{\alpha}$ also for $w \in \partial \mathbb{D}$ such that the function $\alpha$ has an angular derivative in the sense of Carathéodory (an ADC) at $w$. Recall that $\alpha$ is said to have an ADC at $w \in \partial \mathbb{D}$ if there exist complex numbers $\alpha(w)$ and $\alpha^{\prime}(w)$ such that $\alpha(z) \rightarrow \alpha(w) \in \partial \mathbb{D}$ and $\alpha^{\prime}(z) \rightarrow \alpha^{\prime}(w)$ whenever $z$ from $\mathbb{D}$ tends to $w$ non-tangentially (that is, with $|z-w| /(1-|z|)$ bounded). In that case, every $f \in K_{\alpha}$ has a non-tangential limit $f(w)$ at $w$, the functions $k_{w}^{\alpha}$ and $\widetilde{k}_{w}^{\alpha}$ belong to $K_{\alpha}$, and $f(w)=\left\langle f, k_{w}^{\alpha}\right\rangle$ (for more details, see for example [6, Thm. 7.4.1.]).

It was proved in [10] that the only rank-one operators in $\mathscr{T}(\alpha)$ are the scalar multiples of $\widetilde{k}_{w}^{\alpha} \otimes k_{w}^{\alpha}$ and $k_{w}^{\alpha} \otimes \widetilde{k}_{w}^{\alpha}$ for all $w \in \mathbb{D}$ and all $w \in \partial \mathbb{D}$ such that $\alpha$ has an $\mathrm{ADC}$ at $w$.

In the asymmetric case, the authors in [8] showed that the operators $\widetilde{k}_{w}^{\beta} \otimes k_{w}^{\alpha}$ and $k_{w}^{\beta} \otimes \widetilde{k}_{w}^{\alpha}$ belong to $\mathscr{T}(\alpha, \beta)$ for every $w \in \mathbb{D}$ and every $w \in \partial \mathbb{D}$ such that both $\alpha$ and $\beta$ have an ADC at $w$. It is natural to ask: are the scalar multiples of these the only rank-one operators in $\mathscr{T}(\alpha, \beta)$ ? This issue was addressed in [9]. The author in [9] proved that if $K_{\alpha}$ is one dimensional and $K_{\beta}$ has a finite dimension larger than two (or $K_{\beta}$ is one dimensional and $\operatorname{dim} K_{\alpha}>2$ ), then the answer is negative: there exists a rank-one operator in $\mathscr{T}(\alpha, \beta)$ that is neither a scalar multiple of $\widetilde{k}_{w}^{\beta} \otimes k_{w}^{\alpha}$ nor a scalar multiple of $k_{w}^{\beta} \otimes \widetilde{k}_{w}^{\alpha}$. Nevertheless, it was also showed in [9] that the answer is positive for all other finite-dimensional cases (that is, whenever $\alpha$ and $\beta$ are two finite Blaschke products):

Theorem 1.1 ([9, Thm. 4.5]) Let $\alpha$ and $\beta$ be two finite Blaschke products of degree $m>0$ and $n>0$, respectively. The only rank-one operators in $\mathscr{T}(\alpha, \beta)$ are the non-zero scalar multiples of the operators $\widetilde{k}_{w}^{\beta} \otimes k_{w}^{\alpha}$ and $k_{w}^{\beta} \otimes \widetilde{k}_{w}^{\alpha}, w \in \overline{\mathbb{D}}$, if and only if either $m \leq 2$, or $m>1$ and $n>1$.

In this paper, we resolve the issue in the general case. In particular, we prove that if both $K_{\alpha}$ and $K_{\beta}$ have dimension larger than one (and not necessarily finite), then the only rank-one operators in $\mathscr{T}(\alpha, \beta)$ are the non-zero scalar multiples of $\widetilde{k}_{w}^{\beta} \otimes k_{w}^{\alpha}$ and $k_{w}^{\beta} \otimes \widetilde{k}_{w}^{\alpha}$. 


\section{Rank-One Asymmetric Truncated Toeplitz Operators}

In [10], Sarason gave several characterizations of the operators from $\mathscr{T}(\alpha)$. For example, he proved that a bounded linear operator $A$ on $K_{\alpha}$ is a truncated Toeplitz operator if and only if $A-S_{\alpha} A S_{\alpha}^{*}$ is a rank-two operator of a special kind, where $S_{\alpha}=A_{z}^{\alpha}$ is the so-called compressed shift. Similar characterizations of asymmetric truncated Toeplitz operators were given in [2,3] and [9] for some particular cases. Here, we use the following two characterizations of asymmetric truncated Toeplitz operators.

Theorem 2.1 ([7, Thm. 2.1]) Let $A$ be a bounded linear operator from $K_{\alpha}$ into $K_{\beta}$. Then, $A \in \mathscr{T}(\alpha, \beta)$ if and only if there exist $\psi \in K_{\beta}$ and $\chi \in K_{\alpha}$ such that

$$
A-S_{\beta} A S_{\alpha}^{*}=\psi \otimes k_{0}^{\alpha}+k_{0}^{\beta} \otimes \chi
$$

Corollary 2.2 ([7, Cor. 2.7(c)]) Let A be a bounded linear operator from $K_{\alpha}$ into $K_{\beta}$. Then, $A \in \mathscr{T}(\alpha, \beta)$ if and only if $A$ is shift invariant, that is,

$$
\langle A S f, S g\rangle=\langle A f, g\rangle
$$

for all $f \in K_{\alpha}, g \in K_{\beta}$ such that $S f \in K_{\alpha}, S g \in K_{\beta}$.

Note here that if $f \in K_{\alpha}$, then $S f \in K_{\alpha}$ if and only if $f$ is orthogonal to $\widetilde{k}_{0}^{\alpha}$ (see [10, p. 512]).

In his proof of [10, Thm. 5.1], Sarason uses the fact that every operator from $\mathscr{T}(\alpha)$ is $C_{\alpha}$-symmetric. Recall that an operator $A$ on $K_{\alpha}$ is said to be $C_{\alpha}$-symmetric if $C_{\alpha} A C_{\alpha}=A^{*}$. In particular, $C_{\alpha} S_{\alpha} C_{\alpha}=S_{\alpha}^{*}$. In the case of asymmetric truncated Toeplitz operators it is known that $A \in \mathscr{T}(\alpha, \beta)$ if and only if $C_{\beta} A C_{\alpha} \in \mathscr{T}(\alpha, \beta)$ (see [9, p. 9]). So, here we take the approach from [9], that is, we use the following lemma (compare with [9, Lem. 4.4]; see also [10, pp. 503-504]).

Lemma 2.3 Let $\alpha$ and $\beta$ be two inner functions such that $K_{\alpha}$ and $K_{\beta}$ have dimension $m>1$ and $n>1$, respectively. Let $f \in K_{\alpha}, g \in K_{\beta}$ be two non-zero functions such that $g \otimes f$ belongs to $\mathscr{T}(\alpha, \beta)$ and let $w \in \overline{\mathbb{D}}$. Then,

(a) $g$ is a scalar multiple of $k_{w}^{\beta}$ if and only if $f$ is a scalar multiple of $\widetilde{k}_{w}^{\alpha}$,

(b) $g$ is a scalar multiple of $\widetilde{k}_{w}^{\beta}$ if and only if $f$ is a scalar multiple of $k_{w}^{\alpha}$.

Proof Let $f \in K_{\alpha}, g \in K_{\beta}$ be two non-zero functions such that $g \otimes f$ belongs to $\mathscr{T}(\alpha, \beta)$.

We first show that if $g$ is a scalar multiple of $k_{w}^{\beta}$ for some $w \in \overline{\mathbb{D}}$, then $f$ is a scalar multiple of $\widetilde{k}_{w}^{\alpha}$. Actually, without loss of generality we can assume that $g=k_{w}^{\beta}$ for some $w \in \overline{\mathbb{D}}$.

Note here, that for $w \in \partial \mathbb{D}$, we automatically assume that $\beta$ has an $\mathrm{ADC}$ at $w$ (this is implied by the fact that $k_{w}^{\beta} \in K_{\beta}$ ) and we must prove that $\alpha$ also has an ADC at $w$.

We consider two cases. 
Case 1. $\beta(0)=0$.

Since the operator $k_{w}^{\beta} \otimes f$ belongs to $\mathscr{T}(\alpha, \beta)$, it must be shift invariant in the sense of Corollary 2.2. That is,

$$
\left\langle\left(k_{w}^{\beta} \otimes f\right) S h_{\alpha}, S h_{\beta}\right\rangle=\left\langle\left(k_{w}^{\beta} \otimes f\right) h_{\alpha}, h_{\beta}\right\rangle
$$

for all $h_{\alpha} \in K_{\alpha}$ and $h_{\beta} \in K_{\beta}$ such that $S h_{\alpha} \in K_{\alpha}$ and $S h_{\beta} \in K_{\beta}$. Equivalently,

$$
\begin{aligned}
\left\langle\left(k_{w}^{\beta} \otimes f\right) S h_{\alpha}, S h_{\beta}\right\rangle-\left\langle\left(k_{w}^{\beta} \otimes f\right) h_{\alpha}, h_{\beta}\right\rangle & =\left\langle S h_{\alpha}, f\right\rangle\left\langle k_{w}^{\beta}, S h_{\beta}\right\rangle-\left\langle h_{\alpha}, f\right\rangle\left\langle k_{w}^{\beta}, h_{\beta}\right\rangle \\
& =\left\langle h_{\alpha},\left\langle h_{\beta}, S_{\beta}^{*} k_{w}^{\beta}\right\rangle S_{\alpha}^{*} f-\left\langle h_{\beta}, k_{w}^{\beta}\right\rangle f\right\rangle=0
\end{aligned}
$$

for all $h_{\alpha} \in K_{\alpha}$ and $h_{\beta} \in K_{\beta}$, such that $h_{\alpha} \perp \widetilde{k}_{0}^{\alpha}$ and $h_{\beta} \perp \widetilde{k}_{0}^{\beta}$. But it follows from

$$
S_{\beta}^{*} k_{w}^{\beta}=\bar{w} k_{w}^{\beta}-\overline{\beta(w)} \tilde{k}_{0}^{\beta}
$$

(see [10, Lem. 2.2(a)] and its Corollary) that for every $h_{\beta} \perp \widetilde{k}_{0}^{\beta}$, we have

$$
\left\langle h_{\beta}, S_{\beta}^{*} k_{w}^{\beta}\right\rangle=w\left\langle h_{\beta}, k_{w}^{\beta}\right\rangle
$$

Thus, the shift invariance of $k_{w}^{\beta} \otimes f$ means that

$$
\left\langle k_{w}^{\beta}, h_{\beta}\right\rangle\left\langle h_{\alpha}, w S_{\alpha}^{*} f-f\right\rangle=0
$$

for all $h_{\alpha} \perp \widetilde{k}_{0}^{\alpha}$ and $h_{\beta} \perp \widetilde{k}_{0}^{\beta}$.

Since the dimension of $K_{\beta}$ is greater than one, the functions $k_{w}^{\beta}$ and $\widetilde{k}_{0}^{\beta}$ are linearly independent. Indeed, if $k_{w}^{\beta}$ and $\widetilde{k}_{0}^{\beta}$ were linearly dependent, then so would be $\widetilde{k}_{w}^{\beta}$ and $k_{0}^{\beta} \equiv 1$. But, here it is easy to verify that if $\widetilde{k}_{w}^{\beta}=c k_{0}^{\beta}=c$, then $\beta(z)=c z$ and $K_{\beta}$ is one dimensional-a contradiction.

Therefore, there exists a function $h_{\beta} \in K_{\beta}$ orthogonal to $\widetilde{k}_{0}^{\beta}$ and such that $\left\langle k_{w}^{\beta}, h_{\beta}\right\rangle \neq 0$. Putting such $h_{\beta}$ into (2.1), we see that

$$
\left\langle h_{\alpha}, w S_{\alpha}^{*} f-f\right\rangle=0
$$

for all $h_{\alpha} \in K_{\alpha}, h_{\alpha} \perp \widetilde{k}_{0}^{\alpha}$. Thus,

$$
\left(I_{K_{\alpha}}-w S_{\alpha}^{*}\right) f=c \widetilde{k}_{0}^{\alpha}
$$

or, using $C_{\alpha}$-symmetry of $S_{\alpha}$,

$$
\left(I_{K_{\alpha}}-\bar{w} S_{\alpha}\right) C_{\alpha} f=\bar{c} k_{0}^{\alpha}
$$

for some complex number $c$ (here $I_{K_{\alpha}}$ denotes the identity operator on $K_{\alpha}$ ). 
If $c=0$ and $|w|<1$, then, by (2.2) and invertibility of $I_{K_{\alpha}}-\bar{w} S_{\alpha}$, we have $C_{\alpha} f=0$. This implies that $f=0$ which contradicts the assumption that $f$ is non-zero.

If $c=0$ and $|w|=1$, then by (2.2),

$$
S_{\alpha} C_{\alpha} f=w C_{\alpha} f
$$

which again is not possible since $S_{\alpha}$ has no eigenvalues on $\partial \mathbb{D}([10$, Lem. 2.5]).

Therefore, $c \neq 0$ and without loss of generality we can assume that $c=1$. Hence, instead of (2.2) we consider

$$
\left(I_{K_{\alpha}}-\bar{w} S_{\alpha}\right) C_{\alpha} f=k_{0}^{\alpha}
$$

As above, if $|w|<1$, then $I_{K_{\alpha}}-\bar{w} S_{\alpha}$ is invertible. Since $\left(I_{K_{\alpha}}-\bar{w} S_{\alpha}\right) k_{w}^{\alpha}=k_{0}^{\alpha}$ (for $w=0$ this is obvious, for $w \neq 0$ see [10, Lem. 2.2(b)]), we have

$$
f=C_{\alpha}\left(I_{K_{\alpha}}-\bar{w} S_{\alpha}\right)^{-1} k_{0}^{\alpha}=C_{\alpha} k_{w}^{\alpha}=\widetilde{k}_{w}^{\alpha} .
$$

If $|w|=1$, then applying $C_{\alpha}$ to both sides of (2.3) and using $C_{\alpha}$-symmetry of $S_{\alpha}$ we get

$$
f(z)-w \cdot \frac{f(z)-f(0)}{z}=\frac{\alpha(z)-\alpha(0)}{z}, \quad z \in \mathbb{D} .
$$

Hence,

$$
\frac{\alpha(z)-(\alpha(0)+w f(0))}{z-w}=f(z) \in H^{2}
$$

and by [6, Thm. 7.4.1.] $\alpha$ has an ADC at $w$ with the non-tangential limit $\alpha(w)$ equal to $\alpha(0)+w f(0), \tilde{k}_{w}^{\alpha}$ belongs to $K_{\alpha}$ and

$$
f(z)=\frac{\alpha(z)-\alpha(w)}{z-w}=\widetilde{k}_{w}^{\alpha}
$$

This completes the proof in the first case.

Case 2. $\beta(0) \neq 0$.

For any two complex numbers $a, b$ and functions $f \in K_{\alpha}, g \in K_{\beta}$ define

$$
J_{a}^{\alpha} f(z)=\frac{\sqrt{1-|a|^{2}}}{1-\bar{a} \alpha(z)} \cdot f(z) \quad \text { and } \quad J_{b}^{\beta} g(z)=\frac{\sqrt{1-|b|^{2}}}{1-\bar{b} \beta(z)} \cdot g(z)
$$

It is known that $J_{a}^{\alpha}$ is a unitary map from $K_{\alpha}$ onto $K_{\alpha_{a}}$, where

$$
\alpha_{a}(z)=\frac{a-\alpha(z)}{1-\bar{a} \alpha(z)}
$$


and that $J_{b}^{\beta}$ is a unitary map from $K_{\beta}$ onto $K_{\beta_{b}}$, where

$$
\beta_{b}(z)=\frac{b-\beta(z)}{1-\bar{b} \beta(z)}
$$

(see [10, pp. 521-523] for more details). It was also proved in [8] that $A \in \mathscr{T}(\alpha, \beta)$ if and only if $J_{b}^{\beta} A\left(J_{a}^{\alpha}\right)^{-1} \in \mathscr{T}\left(\alpha_{a}, \beta_{b}\right)$ ([8, Prop. 2.5]).

Take $a=0$ and $b=\beta(0)$. Clearly, $\alpha_{0}=\alpha$ and $J_{0}^{\alpha}=I_{K_{\alpha}}$. Therefore, since $k_{w}^{\beta} \otimes f$ belongs to $\mathscr{T}(\alpha, \beta)$, the rank-one operator

$$
J_{b}^{\beta}\left(k_{w}^{\beta} \otimes f\right)\left(J_{a}^{\alpha}\right)^{-1}=\left(J_{b}^{\beta} k_{w}^{\beta}\right) \otimes f
$$

belongs to $\mathscr{T}\left(\alpha, \beta_{b}\right)$. A simple computation reveals that

$$
J_{b}^{\beta} k_{w}^{\beta}=\frac{1-b \overline{\beta(w)}}{\sqrt{1-|b|^{2}}} \cdot k_{w}^{\beta_{b}}
$$

(this can also be seen using Lemma 2.4 from [8]). Hence, the rank-one operator $k_{w}^{\beta_{b}} \otimes f$ belongs to $\mathscr{T}\left(\alpha, \beta_{b}\right)$, and, since $\beta_{b}(0)=0, f$ is a scalar multiple of $\widetilde{k}_{w}^{\alpha}$ by Case 1 .

This completes the proof in the second case and the proof of the first implication in (a).

To prove the first implication in (b) assume that $g=\widetilde{k}_{w}^{\beta}$ for some $w \in \overline{\mathbb{D}}$. Then the rank-one operator

$$
k_{w}^{\beta} \otimes C_{\alpha} f=C_{\beta}(g \otimes f) C_{\alpha}
$$

belongs to $\mathscr{T}(\alpha, \beta)$ [9, p. 9], and it follows from the part of (a) proved above that $C_{\alpha} f$ is a scalar multiple of $\widetilde{k}_{w}^{\alpha}$ and $f$ is a scalar multiple of $k_{w}^{\alpha}$.

To complete the proof of (a) assume that $f=\widetilde{k}_{w}^{\alpha}$. Then,

$$
\widetilde{k}_{w}^{\alpha} \otimes g=\left(g \otimes \widetilde{k}_{w}^{\alpha}\right)^{*}
$$

belongs to $\mathscr{T}(\beta, \alpha)$ [2, Lem. 3.2], and it follows from the part of (b) proved above that $g$ is a scalar multiple of $k_{w}^{\beta}$.

Similarly, if $f=k_{w}^{\alpha}$, then

$$
k_{w}^{\alpha} \otimes g=\left(g \otimes k_{w}^{\alpha}\right)^{*}
$$

belongs to $\mathscr{T}(\beta, \alpha)$ and it follows from (a) that $g$ is a scalar multiple of $\widetilde{k}_{w}^{\beta}$. This completes the proof of the lemma.

Using Lemma 2.3, one can modify the proof of [10, Thm. 5.1(c)] to show the following. 
Theorem 2.4 Let $\alpha$ and $\beta$ be two inner functions such that $K_{\alpha}$ and $K_{\beta}$ have dimension $m>1$ and $n>1$, respectively. Then the only rank-one operators in $\mathscr{T}(\alpha, \beta)$ are the non-zero scalar multiples of the operators $\widetilde{k}_{w}^{\beta} \otimes k_{w}^{\alpha}$ and $k_{w}^{\beta} \otimes \widetilde{k}_{w}^{\alpha}$ where $w \in \mathbb{D}$ or $w \in \partial \mathbb{D}$ and $\alpha$ and $\beta$ have an $A D C$ at $w$.

Note that neither the above nor [9] includes the case when one of the model spaces is one dimensional and the other is infinite dimensional.

Let us now assume that $\alpha, \beta$ are two inner functions such that $\operatorname{dim} K_{\alpha}=1$ and $\operatorname{dim} K_{\beta}=+\infty$.

Clearly, every bounded linear operator $A$ from $K_{\alpha}$ into $K_{\beta}$ is of rank one. Moreover, since $K_{\alpha}$ is spanned by $k_{0}^{\alpha}, A-S_{\beta} A S_{\alpha}^{*}$ is a rank-one operator of the form $\psi \otimes k_{0}^{\alpha}$ for some $\psi \in K_{\beta}$. So here, by Theorem 2.1, all bounded linear operators from $K_{\alpha}$ into $K_{\beta}$ are asymmetric truncated Toeplitz operators of rank one.

As the finite-dimensional case suggests, one can expect that here there are more rank-one operators in $\mathscr{T}(\alpha, \beta)$ than just scalar multiples of $\widetilde{k}_{w}^{\beta} \otimes k_{w}^{\alpha}$ and $k_{w}^{\beta} \otimes \widetilde{k}_{w}^{\alpha}$. We now prove that this is indeed the case. We do this in two steps: (1) we show that if every rank-one operator from $\mathscr{T}(\alpha, \beta)$ is a scalar multiple of $\widetilde{k}_{w}^{\beta} \otimes k_{w}^{\alpha}$ or $k_{w}^{\beta} \otimes \widetilde{k}_{w}^{\alpha}$ for some $w \in \overline{\mathbb{D}}$, then every non-zero function from $K_{\beta}$ must be a scalar multiple of a reproducing kernel or a conjugate kernel; (2) we prove that since $\operatorname{dim} K_{\beta}=+\infty$, there must exist $f \in K_{\beta}$ that is neither a scalar multiple of a reproducing kernel nor a scalar multiple of a conjugate kernel.

Step 1. Let $f \in K_{\beta} \backslash\{0\}$. Then, $f \otimes k_{0}^{\alpha} \in \mathscr{T}(\alpha, \beta)$ and there exist $w \in \overline{\mathbb{D}}$ and $c \neq 0$ such that

$$
f \otimes k_{0}^{\alpha}=c\left(\widetilde{k}_{w}^{\beta} \otimes k_{w}^{\alpha}\right) \quad \text { or } \quad f \otimes k_{0}^{\alpha}=c\left(k_{w}^{\beta} \otimes \widetilde{k}_{w}^{\alpha}\right) .
$$

Since $\operatorname{dim} K_{\alpha}=1$, we can replace $k_{0}^{\alpha}$ in the above with $k_{w}^{\alpha}$ or $\widetilde{k}_{w}^{\alpha}$ (multiplying both sides of the assumed equality by a constant if necessary). Then it follows easily that $f$ is a scalar multiple of either $\widetilde{k}_{w}^{\beta}$ or $k_{w}^{\beta}$.

Step 2. Here, we use [1, Prop. 2.8]. Fix $w_{1}, w_{2} \in \mathbb{D}, w_{1} \neq w_{2}$ and let $f=k_{w_{1}}^{\beta}+k_{w_{2}}^{\beta} \in K_{\beta} \backslash\{0\}$. Since $\operatorname{dim} K_{\beta}=+\infty$, by [1, Prop. 2.8], the functions $k_{w_{1}}^{\beta}$, $k_{w_{2}}^{\beta}$ and $\widetilde{k}_{w}^{\beta}$ are linearly independent for any fixed $w \in \mathbb{D}$ and for any fixed $w \in \partial \mathbb{D}$ such that $\beta$ has an ADC at $w$. Thus, $f$ cannot be a scalar multiple of $\widetilde{k}_{w}^{\beta}$. A similar reasoning shows that $f$ cannot be a scalar multiple of $k_{w}^{\beta}$ for any $w \in \overline{\mathbb{D}}$.

We proved that if $\operatorname{dim} K_{\alpha}=1$ and $\operatorname{dim} K_{\beta}=+\infty$, then there is a rank-one operator from $\mathscr{T}(\alpha, \beta)$ that is neither a scalar multiple of $\widetilde{k}_{w}^{\beta} \otimes k_{w}^{\alpha}$ nor a scalar multiple of $k_{w}^{\beta} \otimes \widetilde{k}_{w}^{\alpha}$. The case $\operatorname{dim} K_{\alpha}=+\infty$ and $\operatorname{dim} K_{\beta}=1$ can be treated analogously.

Theorem 2.5 Let $\alpha$ and $\beta$ be two inner functions such that $\operatorname{dim} K_{\alpha}=m$ and $\operatorname{dim} K_{\beta}=n$ (with $m$ or $n$ possibly infinite). The only rank-one operators in $\mathscr{T}(\alpha, \beta)$ are the non-zero scalar multiples of the operators $\widetilde{k}_{w}^{\beta} \otimes k_{w}^{\alpha}$ and $k_{w}^{\beta} \otimes \widetilde{k}_{w}^{\alpha}, w \in \overline{\mathbb{D}}$, if and only if either $m n \leq 2$, or $m>1$ and $n>1$.

Proof Assume that the only rank-one operators in $\mathscr{T}(\alpha, \beta)$ are the non-zero scalar multiples of the operators $\widetilde{k}_{w}^{\beta} \otimes k_{w}^{\alpha}$ and $k_{w}^{\beta} \otimes \widetilde{k}_{w}^{\alpha}$. If both $m$ and $n$ are finite, then $\alpha$ 
and $\beta$ are two finite Blaschke products of degree $m$ and $n$, respectively, and the claim follows from Theorem 1.1. If $m$ or $n$ is infinite, then $m>1$ and $n>1$ as already observed before.

To complete the proof, assume that either $m n \leq 2$, or $m>1$ and $n>1$. If both $m$ and $n$ are finite, then the only rank-one operators in $\mathscr{T}(\alpha, \beta)$ are the non-zero scalar multiples of the operators $\widetilde{k}_{w}^{\beta} \otimes k_{w}^{\alpha}$ and $k_{w}^{\beta} \otimes \widetilde{k}_{w}^{\alpha}$ by Theorem 1.1. If $m$ or $n$ is infinite, then clearly $m>1$ and $n>1$ and the claim follows from Theorem 2.4.

Open Access This article is distributed under the terms of the Creative Commons Attribution 4.0 International License (http://creativecommons.org/licenses/by/4.0/), which permits unrestricted use, distribution, and reproduction in any medium, provided you give appropriate credit to the original author(s) and the source, provide a link to the Creative Commons license, and indicate if changes were made.

\section{References}

1. Bessonov, R.V.: Truncated Toeplitz operators of finite rank. Proc. Am. Math. Soc. 142(4), 1301-1313 (2014)

2. Câmara, C., Jurasik, J., Kliś-Garlicka, K., Ptak, M.: Characterizations of asymmetric truncated Toeplitz operators. Banach J. Math. Anal. 11(4), 899-922 (2017)

3. Câmara, C., Kliś-Garlicka, K., Ptak, M.: Shift invariance and reflexivity of asymmetric truncated Toeplitz operators (preprint)

4. Câmara, M.C., Partington, J.R.: Asymmetric truncated Toeplitz operators and Toeplitz operators with matrix symbol. J. Oper. Theory 77(2), 455-479 (2017)

5. Câmara, M.C., Partington, J.R.: Spectral properties of truncated Toeplitz operators by equivalence after extension. J. Math. Anal. Appl. 433(2), 762-784 (2016)

6. Garcia, S.R., Mashreghi, J.E., Ross, W.: Introduction to Model Spaces and their Operators, Cambridge Studies in Advanced Mathematics, vol. 148. Cambridge University Press, Cambridge (2016)

7. Gu, C., Łanucha, B., Michalska, M.: Characterizations of asymmetric truncated Toeplitz and Hankel operators (preprint)

8. Jurasik, J., Łanucha, B.: Asymmetric truncated Toeplitz operators equal to the zero operator. Ann. Univ. Mariae Curie Skłodowska Sect. A 70(2), 51-62 (2016)

9. Łanucha, B.: On rank-one asymmetric truncated Toeplitz operators on finite-dimensional model spaces. J. Math. Anal. Appl. 454(2), 961-980 (2017)

10. Sarason, D.: Algebraic properties of truncated Toeplitz operators. Oper. Matrices 1(4), 491-526 (2007) 